\title{
The importance of antiandrogen in prostate cancer treatment
}

\author{
Camille Lanz ${ }^{1}$, Mostefa Bennamoun ${ }^{2}$, Petr Macek ${ }^{1}$, Xavier Cathelineau ${ }^{1}$, Rafael Sanchez-Salas ${ }^{1}$ \\ ${ }^{1}$ Department of Urology, ${ }^{2}$ Department of Oncology, Mutualist Montsouris Institute, 42 Boulevard Jourdan, Paris, France \\ Correspondence to: Rafael Sanchez-Salas, MD. Department of Urology, Mutualist Montsouris Institute, 42 Boulevard Jourdan, 75014 Paris, France. \\ Email: raersas@gmail.com; rafael.sanchez-salas@imm.fr. \\ Provenance: This is an article commissioned by the Guest Section Editor Weijun Jiang (Nanjing Normal University, Department of Reproductive and \\ Genetics, Institute of Laboratory Medicine, Jinling Hospital, Nanjing University School of Medicine, Nanjing, China). \\ Comment on: Chi KN, Agarwal N, Bjartell A, et al. Apalutamide for metastatic, castration-sensitive prostate cancer. N Engl J Med 2019;381:13-24.
}

Submitted Aug 09, 2019. Accepted for publication Sep 05, 2019.

doi: $10.21037 / \mathrm{atm} .2019 .09 .53$

View this article at: http://dx.doi.org/10.21037/atm.2019.09.53

\section{The importance of antiandrogen in prostate cancer treatment}

Since 2011, Docetaxel is no longer the exclusive treatment for castration-resistant prostate cancer (CRPC), with the emergence of a new generation of hormonal treatments (1-4). Although the first antiandrogens generation has shown clinical benefit, their low affinity for the androgen receptor (AR) allows for the $5 \%$ to $10 \%$ of free dihydrotestosterone in the prostate to activate the AR (1). Antiandrogens, such as enzalutamide and apalutamide, have nowadays a major role in CRPC treatment. Indeed, enzalutamide has shown an overall survival (OS) improvement in metastatic castration-resistant prostate cancer (mCRPC), before and after chemotherapy (PREVAIL and AFFIRM trials respectively) $(5,6)$, and a metastatic-free survival (MFS) improvement in nonmetastatic castration-resistant prostate cancer (M0CRPC) (PROSPER trial) (7). Subsequently, SPARTAN (Selective Prostate Androgen Receptor Targeting with ARN-509), a randomized placebo-controlled Phase 3 trial, evaluated the benefits of apalutamide on MFS in men with MOCRPC, with all treated by androgen deprivation therapy (ADT), and at a high risk of developing metastases, as defined by a doubling of the prostate-specific antigen time (PSA-DT) of 10 months or less. Its results reported on an improvement in MFS with apalutamide in MOCRPC (8).

\section{Place of AR in prostate cancer treatment}

Huggins and Hodges established the sensitivity to androgen in prostate cancer by observing that a low circulating androgen level could reduce the advanced prostate cancer symptoms (9). The discovery of ARs in the late 1960s led to the understanding that their activations could induce the translocation from the cytoplasm to the nucleus, and thus the expression of proliferation genes. The ADT, by surgical or medical castration, delays this proliferation for a limited period, until the PSA level rises, suggesting a disease progression into a castration-resistance (1). Whereas the disease becomes refractory to the gonadotropinreleasing hormone (GnRH) axis inhibition, the observed response to hormonal manipulations with other agents, such as ketoconazole or diethylstilbestrol, suggests the existence of another pathway (1). The significant levels of androgen in the prostate cancer cells, even though the circulating testosterone level remains at a $95 \%$ decrease, as observed by Labrie in the 1980s (10), is probably provided by the transformation of dehydroepiandrosterone from the adrenal origin into testosterone in the peripheral tissues intracrinology function. This emphasizes that the androgen signal could represent a major role in the disease control. According to Crawford, an optimized androgenic blockage by combining $\mathrm{ADT}$ and antiandrogen at the initiation of ADT, or after the disease progression, could improve the treatment effectiveness (11). The latter has led to the emergence of treatments using competitive ligands for AR.

\section{Place of antiandrogen in prostate cancer}

The AR inhibitors compete with the endogenous androgens for the AR ligand-biding domain. They block the androgen signal by inhibiting the nuclear translocation. The first 
steroidal antiandrogens have given way to safer and more specific nonsteroidal antiandrogens (1). The first generation that was composed by flutamide and nilutamide when combined to ADT, improves the OS between 3 to 8 months, and extends the progression-free survival (PFS) of metastatic castration-sensitive prostate cancer (m1CSPC) (16.5 months with flutamide vs. 13.9 months with placebo, $\mathrm{P}=0.039$; and 20.8 months with nilutamide and 14.9 months with placebo, $\mathrm{P}=0.005)(1,11,12)$. The second generation, bicalutamide, had a relatively modest clinical benefit since when alone, no difference with the standard of care has been underlined on the survival in men with locally advanced and metastatic disease $(1,13,14)$. According to the Early Prostate Cancer (EPC) trial program that evaluated bicalutamide in a localized or locally advanced disease, bicalutamide improved the PFS of patients with a locally advanced disease, especially for those who had undergone radiotherapy $(\mathrm{P}=0.0031)$ $(1,13)$. The new antiandrogen generation (enzalutamide, apalutamide, darolutamide), with a higher affinity to the AR, optimizes the androgen blockade effect. Enzalutamide was the first approved antiandrogen by the FDA in 2012 . It has a five to eightfold higher affinity for the AR than bicalutamide. Its clinical efficiency was verified in mCRPC ( $81 \%$ and $29 \%$ reduction in the risk of radiographic progression and death, respectively, with enzalutamide and $\mathrm{ADT})$, and in MOCRPC (71\% lower risk of metastasis or death than placebo) $(6,7,15-17)$. Darolutamide is currently evaluated in m1CSPC when added to ADT and docetaxel in ARASENS (NCT02799602), in M0CRPC in ARAMIS (NCT02200614), and in mCRPC in ARASENS (NCT02799602).

\section{Apalutamide}

Apalutamide is an oral third-generation nonsteroidal AR inhibitor that was discovered in 2007 and approved by the FDA in 2018. It is closely related to enzalutamide with a similar mechanism of action. Its affinity to AR is five to tenfold higher than bicalutamide. Unlike enzalutamide, apalutamide does not cross the blood-brain barrier and has a greater antitumor activity at a lower dose.

Different studies have reported that apalutamide could reduce the PSA level from $46 \%$ to $89 \%$ in mCRPC and in MOCRPC $(18,19)$. SPARTAN observed an MFS improvement with apalutamide when compared to a placebo (40.5 and 16.2 months, respectively, hazard ratio (HR) 0.28; $\mathrm{P}<0.001)$ in M0CRPC, and with a significantly longer time to the symptomatic progression with apalutamide (HR, $0.45)(8)$. Since then, apalutamide has been recommended for the treatment of patients with MOCRPC (8). It has generated 5 trials: at an earlier stage as in low risk prostate cancer (Active Surveillance with or without Apalutamide Treatment in Low Risk Prostate Cancer, NCT03088124); localized high risk disease (ATLAS, NCT02531516); biochemical recurrence (EMBARK, NCT02319837), and m1CSPC (TITAN, NCT02489318) (20); as well as at a more advanced stage as mCRPC (ACIS, NCT02257736).

\section{Place of apalutamide in international guidelines and the standard of care in patients with m1CSPC in 2019}

Among the EAU, NCCN, AUA, and AFU2019 guidelines, the place of apalutamide mainly concerns patients with MOCRPC, who are at a high risk of metastases (as assessed by a PSA-DT $\leq 10$ months). They all recommend combining a new antiandrogen, with either apalutamide or enzalutamide, with ADT.

In the case of m1CSPC, they all recommend introducing an ADT to palliate the symptoms and reduce the risk of metastasis complications. While the guidelines are unclear for a low risk and volume because of a lack of evidence, they all advise combining ADT to an adjuvant therapy, such as docetaxel, for the high-volume disease, and abiraterone with prednisone for the high-risk ones. According to CHAARTED (which evaluated docetaxel when combined with $\mathrm{ADT}$ versus $\mathrm{ADT}$ alone in m1CSPC), a high metastases volume represents at least 4 bone metastases, including 1 outside the axial skeleton, or visceral metastasis (21). According to LATITUDE (which evaluated abiraterone plus prednisone when combined to ADT versus ADT alone in m1CSPC), a high-risk disease included at least 2 high-risk features ( $\geq 3$ bone metastasis, visceral metastasis, and a grade $\geq 4$ ISUP score) (22).

\section{Apalutamide for metastatic, castration-sensitive prostate cancer-TITAN}

The Targeted Investigational Treatment Analysis of Novel Anti-androgen trial (TITAN) that was published in May 2019 in the New England Journal of Medicine was a double-blind Phase 3 randomized trial designed by Chi et al. They aimed to assess the benefits of apalutamide $(240 \mathrm{mg}$ per day) when compared to a placebo, with both of them in a combination with $\mathrm{ADT}$, on $\mathrm{OS}$ and radiographic 
progression-free survival (RFPS) in 1,052 patients with m1CSPC, while at the same time, maintaining a reasonable health-related quality of life. After a median follow-up of 22.4 months, TITAN reported a significant improvement in RFPS and OS at 24 months in the apalutamide group [68.2\% in the apalutamide group vs. $47.5 \%$ in the placebo group, HR $0.48,95 \%$ confidence interval (CI), 0.39 to 0.60 ; $\mathrm{P}<0.001$; and $82.4 \%$ in the apalutamide group vs. $73.5 \%$ in the placebo group, HR $0.67 ; 95 \% \mathrm{CI}, 0.51$ to $0.89 ; \mathrm{P}=0.005$, respectively], with a quality of life preservation.

\section{Radiographic progression-free survival (RPFS)}

TITAN was the first Phase 3 trial comparing the benefits of apalutamide upon m1CSPC, whatever the metastasis volume. As well as the equivalent clinical trials which evaluated $\mathrm{ADT}$ when associated with either enzalutamide or a placebo (ARCHES - NCT02677896 - results presented at ASCO-GU 2019), or ADT when associated with either enzalutamide or an older generation nonsteroidal antiandrogen (ENZAMET - NCT02446405 - results presented at ASCO-GU 2019) (Table 1), TITAN reported a real RPFS improvement at 24 months $(68.2 \%$ in the apalutamide group and $47.5 \%$ in the placebo group; HR $0.48 ; 0.39$ to $0.60 ; \mathrm{P}<0.001$ ), leading to a $52 \%$ lower risk of radiographic progression or death in the apalutamide group. This result was really close to the $53 \%$ lower risk of radiographic progression or death in the abiraterone group (HR, $0.47 ; 0.39$ to $0.55 ; \mathrm{P}<0.001$ ), as reported by LATITUDE on m1CSPC (Table 1) (22). This supported the hypothesis that androgen signal inhibition could significantly delay the disease progression and should probably have a place at an earlier stage of the disease.

Furthermore, this benefit of apalutamide remains favorable in the different subgroups analyzed (previous docetaxel, high or low disease volume). In fact, ARCHES and ENZAMET reported on the same conclusion with enzalutamide. However, probably because of a high proportion of the $40 \%$ patients who received a prior therapy with docetaxel in ENZAMET, the clinical PFSs were lower, but they were significant in the different subgroups. It is not clear whether this needs a longer follow-up, or if it is the consequence of the prior treatment.

\section{Overall survival (OS) (Figure 1)}

The major strength of TITAN was to identify a significant favorable benefit on OS since $82.4 \%$ of the patients were alive at 24 months in the apalutamide group and only $73.5 \%$ in the placebo group (HR for death, $0.67 ; 0.51$ to 0.89; $\mathrm{P}=0.005)$. Thus, apalutamide provides a $33 \%$ lower risk of death. This reflects, in particular, on the advantages of apalutamide, since those patients with a high volume m1CSPC did not consistently receive the gold standard treatment, docetaxel or abiraterone associated with ADT, but they still presented a longer OS (HR 0.68; 0.50-0.92) (20). This is consistent with the significant $38 \%$ lower relative risk of death in patients from the abiraterone group as reported in LATITUDE, despite its population heterogeneity (Table 1, Figure 1), and it underlined that by improving the androgen signal inhibition, it could improve patients with m1CSPC survival (22). Nowadays, the results on OS for enzalutamide are insufficient. While ENZAMET had also observed an improvement in OS when treated with enzalutamide at 3 years (Table 1, Figure 1), ARCHES' OS data are so far immature, but it tended to be favorable to enzalutamide. As well as being significant, the OS benefit in TITAN occurs earlier with apalutamide, after 2 years of treatment.

In addition, the apalutamide benefit remains significant, whatever the disease volume, while the effect of enzalutamide in the stratified groups of ENZAMET appeared to be less consistent and not significant, notably in the high volume disease. Finally, TITAN's OS results for patients who received a prior therapy with docetaxel tended to be favorable, but they were not significant (HR 1.27), as ENZAMET had described it for enzalutamide.

According to TITAN, apalutamide could improve patients with an m1CSPC survival, whatever the disease volume. This led the investigators to unblind the trial, which was really unclear with enzalutamide. However, the best treatment for patients who have already received docetaxel remains unclear.

\section{Adverse event-quality of life}

Just as SPARTAN had on a low discontinuation level due to apalutamide adverse events in $\mathrm{mCRPC}$ without a significant difference $(10.6 \%$ in the apalutamide group and $7.0 \%$ in the placebo group), TITAN observed a lower rate of $8 \%$ of interrupted treatments by apalutamide because of adverse events, with $5.3 \%$ in the placebo group. Furthermore, TITAN observed a lower rate of grade 3 or 4 events and a lower difference between groups than did SPARTAN (42.2\% and $45.1 \%$ in the apalutamide groups, and $40.8 \%$ and $34.2 \%$ in the placebo groups, respectively), as well 


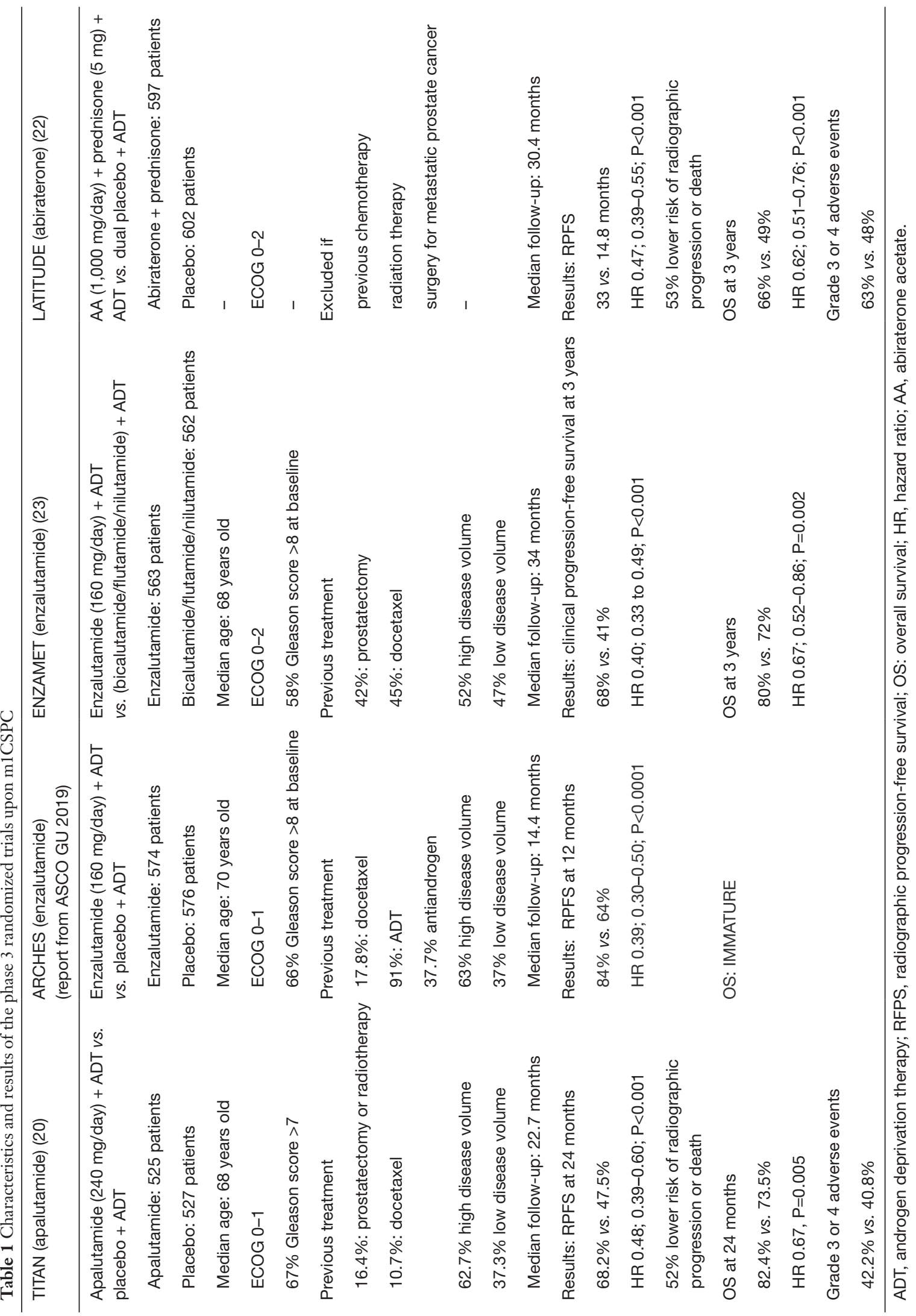


A

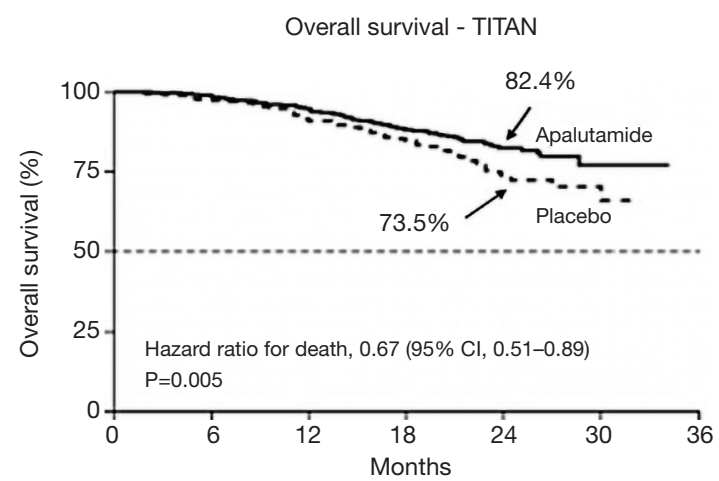

B

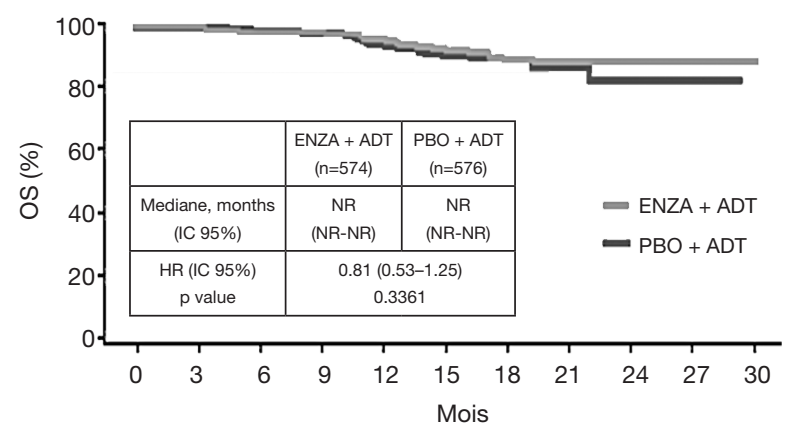

\begin{tabular}{|c|c|c|c|c|c|c|c|c|c|c|c|c|c|}
\hline No. at risk & & & & & & & & & & & & & \\
\hline Apalutamic & 525 & 513 & 490 & 410 & 165 & 14 & 0 & ENZA + ADT & 574 & 559 & 379 & 130 & 13 \\
\hline Placebo & 527 & 509 & 473 & 387 & 142 & 16 & 0 & $P B O+A D T$ & 576 & 548 & 453 & 116 & 5 \\
\hline
\end{tabular}

C

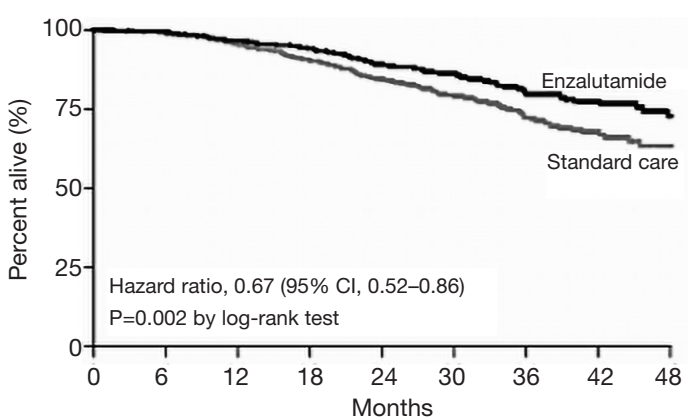

No. at risk

$\begin{array}{llllllllll}\text { Enzalutamide } & 563 & 558 & 541 & 527 & 480 & 340 & 189 & 106 & 45\end{array}$

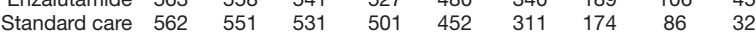

D

Overall survival - LATITUDE

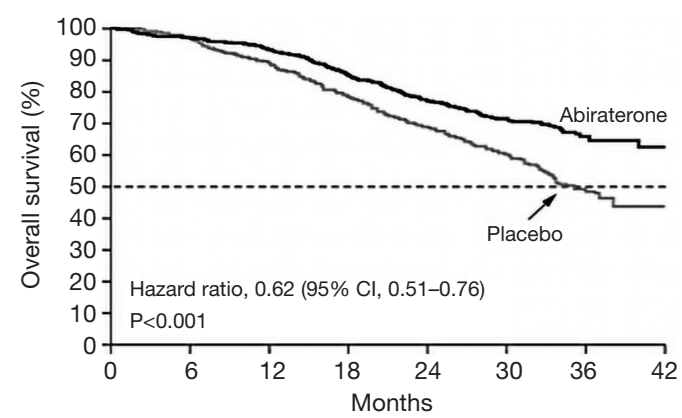

No. at risk

$\begin{array}{lllllllll}\text { Abiraterone } & 597 & 565 & 529 & 479 & 388 & 233 & 93 & 9\end{array}$

Figure 1 Overall survival. Survival curve from: TITAN trial - ENZAMET trial - LATITUDE trial - The new England fournal of Medicine. ARCHES trial - Report from ASCO GU 2019 (20,22,23). ADT, androgen deprivation therapy; NR, not reached; ENZA, enzalutamide; PBO, Placebo.

as a lower serious adverse events rate $(19.8 \%$ and $24.8 \%$, respectively), and an identical death rate due to apalutamide $(8,20)$. Thus, both TITAN and SPARTAN have suggested that apalutamide provides a good quality of life, especially in TITAN, in which the best tolerance to apalutamide was probably due to patients with an early-stage disease and an ECOG score of between 0 and 1 . That raises the matter of vulnerable patient treatments options.

The health-related quality of life in TITAN was evaluated thanks to changes from the baseline in the The Functional Assessment of Cancer Therapy-Prostate Questionnaire (FACT-P), and this seemed to be preserved in both groups. According to LATITUDE, a better AR signal inhibition also improved the quality of life. Indeed, despite $12 \%$ discontinuation of abiraterone due to adverse events (22), the median time to deterioration in functional status, assessed by the same tool, was longer in the abiraterone group than in the placebo group (12.9 and 8.3 months respectively, HR $0.85 ; \mathrm{P}=0.032$ ). The addition of abiraterone and prednisone would therefore appear to delay the progression of pain, fatigue and also offer a good quality of life (24), despite a higher treatment interruption rate than apalutamide. A trial comparing apalutamide and abiraterone could evaluate which one provides a better quality of life.

Using the same FACT-P tool for enzalutamide, ARCHES' data, so far immature, tended toward a preserved quality of life but with no difference between the groups over the length of the study. However, ENZAMET identified $12 \%$ of discontinuation for other reasons than disease progression, and more 1 to 3 adverse events, including fatigue and the risk of seizure, and even more so, when the patients had already been treated by docetaxel. It is nevertheless difficult to identify if the toxic effects corresponded to enzalutamide alone, or to docetaxel (24). 
According to this result, apalutamide is the only antiandrogen that underlined a significant improvement on OS and RPFS in m1CSPC, while keeping a good quality of life.

\section{Conclusions}

$\mathrm{ADT}$ alone is insufficient for the management of metastatic prostate cancer since most patients with m1CSPC will progress to the castration-resistant disease within 1 year (25). TITAN confirms LATITUDE's hypothesis, suggesting that a stronger AR signaling inhibition is required to improve patients with m1CSPC outcomes. Apalutamide is currently the only antiandrogen that has shown a sooner real benefit in OS after 2 years of treatment, with an acceptable tolerance (20). This could change the management of m1CSPC and help to choose between the different antiandrogens. This benefit is observed at different stages of the disease (resistance to castration, regardless of the disease volume, "de novo" metastatic disease or metastatic recurrence of a disease initially localized at diagnosis) and raises the question of the potential benefit of earlier introduction of antiandrogens to improve disease outcomes. Due to the lack of trials comparing the AR inhibitors between each other, one would probably have to choose the antiandrogen according to the co-morbidity and general state and probably to the cost.

However, it remains difficult to precisely define the optimal time to begin these new antiandrogens, especially when these patients had already received docetaxel. Their usefulness in mCRPC, M0CRPC and m1CSPC is currently demonstrated. Indeed, the lack of survival results in patients who have previously received apalutamide and the fact that only $38 \%$ of patients in the apalutamide group received subsequent post-progression treatment may raise doubts about the appropriate time to start apalutamide in the disease evolution. One may wonder if the disease response to subsequent post-progression treatments could be optimized by taking previous apalutamide. Ongoing clinical trials are attempting to assess whether the introduction of these new antiandrogens before the emergence of metastases can delay the progression of the disease, particularly at the stage of low-risk localized diseases. This raises the issue of tolerance as well as the determination of criteria for selecting patients who could benefit from it.

\section{Acknowledgments}

None.

\section{Footnote}

Conflicts of Interest: The authors have no conflicts of interest to declare.

Ethical Statement: The authors are accountable for all aspects of the work in ensuring that questions related to the accuracy or integrity of any part of the work are appropriately investigated and resolved.

\section{References}

1. Crawford ED, Schellhammer PF, McLeod DG, et al. Androgen receptor targeted treatments of prostate cancer: 35 years of progress with antiandrogens. J Urol 2018;200:956-66.

2. de Bono JS, Logothetis CJ, Molina A, et al. Abiraterone and increased survival in metastatic prostate cancer. $\mathrm{N}$ Engl J Med 2011;364:1995-2005.

3. Petrylak DP, Tangen CM, Hussain MHA, et al. Docetaxel and estramustine compared with mitoxantrone and prednisone for advanced refractory prostate cancer. $\mathrm{N}$ Engl J Med 2004;351:1513-20.

4. Tannock IF, de Wit R, Berry WR, et al. Docetaxel plus prednisone or mitoxantrone plus prednisone for advanced prostate cancer. N Engl J Med 2004;351:1502-12.

5. Scher HI, Fizazi K, Saad F, et al. Increased survival with enzalutamide in prostate cancer after chemotherapy. $\mathrm{N}$ Engl J Med 2012;367:1187-97.

6. Beer TM, Armstrong AJ, Rathkopf DE, et al. Enzalutamide in metastatic prostate cancer before chemotherapy. N Engl J Med 2014;371:424-33.

7. Hussain M, Fizazi K, Saad F, et al. Enzalutamide in men with nonmetastatic, castration-resistant prostate cancer. $\mathrm{N}$ Engl J Med 2018;378:2465-74.

8. Smith MR, Saad F, Chowdhury S, et al. Apalutamide treatment and metastasis-free survival in prostate cancer. N Engl J Med 2018;378:1408-18.

9. Huggins C, Hodges CV. Studies on prostatic cancer. I. The effect of castration, of estrogen and androgen injection on serum phosphatases in metastatic carcinoma of the prostate. CA Cancer J Clin 1972;22:232-40.

10. Labrie F, Dupont A, Belanger A. Complete androgen blockade for the treatment of prostate cancer. Important Adv Oncol 1985;193-217.

11. Crawford ED, Eisenberger MA, McLeod DG, et al. A controlled trial of leuprolide with and without flutamide in prostatic carcinoma. N Engl J Med 1989;321:419-24. 
12. Janknegt RA, Abbou CC, Bartoletti R, et al. Orchiectomy and nilutamide or placebo as treatment of metastatic prostatic cancer in a multinational double-blind randomized trial. J Urol 1993;149:77-82; discussion 83.

13. Iversen P, McLeod DG, See WA, et al. Antiandrogen monotherapy in patients with localized or locally advanced prostate cancer: final results from the bicalutamide Early Prostate Cancer programme at a median follow-up of 9.7 years. BJU Int 2010;105:1074-81.

14. Iversen P, Tyrrell CJ, Kaisary AV, et al. Bicalutamide monotherapy compared with castration in patients with nonmetastatic locally advanced prostate cancer: 6.3 years of followup. J Urol 2000;164:1579-82.

15. Siemens DR, Klotz L, Heidenreich A, et al. Efficacy and safety of enzalutamide vs bicalutamide in younger and older patients with metastatic castration resistant prostate cancer in the TERRAIN trial. J Urol 2018;199:147-54.

16. Merseburger AS, Scher HI, Bellmunt J, et al. Enzalutamide in European and North American men participating in the AFFIRM trial. BJU Int 2015;115:41-9.

17. Penson DF, Armstrong AJ, Concepcion R, et al. Enzalutamide versus bicalutamide in castrationresistant prostate cancer: the STRIVE trial. J Clin Oncol 2016;34:2098-106.

18. Rathkopf DE, Morris MJ, Fox JJ, et al. Phase I study of ARN-509, a novel antiandrogen, in the treatment of castration-resistant prostate cancer. J Clin Oncol 2013;31:3525-30.

Cite this article as: Lanz C, Bennamoun M, Macek P, Cathelineau X, Sanchez-Salas R. The importance of antiandrogen in prostate cancer treatment. Ann Transl Med 2019;7(Suppl 8):S362. doi: 10.21037/atm.2019.09.53
19. Smith MR, Antonarakis ES, Ryan CJ, et al. Phase 2 Study of the safety and antitumor activity of apalutamide (ARN509), a potent androgen receptor antagonist, in the highrisk nonmetastatic castration-resistant prostate cancer cohort. Eur Urol 2016;70:963-70.

20. Chi KN, Agarwal N, Bjartell A, et al. Apalutamide for metastatic, castration-sensitive prostate cancer. N Engl J Med 2019;381:13-24.

21. Sweeney CJ, Chen YH, Carducci M, et al. Chemohormonal therapy in metastatic hormone-sensitive prostate cancer. N Engl J Med 2015;373:737-46.

22. Fizazi K, Tran N, Fein L, et al. Abiraterone plus prednisone in metastatic, castration-sensitive prostate cancer. N Engl J Med 2017;377:352-60.

23. Davis ID, Martin AJ, Stockler MR, et al. Enzalutamide with standard first-line therapy in metastatic prostate cancer. N Engl J Med 2019;381:121-31.

24. Chi KN, Protheroe A, Rodríguez-Antolín A, et al. Patient-reported outcomes following abiraterone acetate plus prednisone added to androgen deprivation therapy in patients with newly diagnosed metastatic castrationnaive prostate cancer (LATITUDE): an international, randomised phase 3 trial. Lancet Oncol 2018;19:194-206.

25. Gillessen S, Attard G, Beer TM, et al. Management of patients with advanced prostate cancer: the report of the advanced prostate cancer consensus conference APCCC 2017. Eur Urol 2018;73:178-211. 